Supporting Information

\title{
Platelet 3D preservation using a novel biomimetic nanofiber peptide for reduced apoptosis and easy storage
}

Jiayao Li ${ }^{1,2}$, Lei Wang ${ }^{1, \$}$, Xiaoyang Yi ${ }^{1, \$}$, Yuyuan Ma ${ }^{1}$, Kun Liu ${ }^{1,2}$, Minxia Liu ${ }^{1}$, Shaoduo Yan ${ }^{1}$, Zhenzhu Sun ${ }^{1}$, Yanhong $\mathrm{Li}^{1}$, Ang Lv ${ }^{1}$, Yunfeng Sun ${ }^{1,2}$, Hailong Zhuo ${ }^{3}$, Ying Han ${ }^{1}$, Donggen Wang 1, Jun Liang ${ }^{2 *}$, Qiuxia Fu ${ }^{1, *}$

1 Institute of Health Service and Transfusion Medicine, Academy of Military Medical Sciences, 27(1) Taiping Road, Beijing, 100850, China

2 State Key Laboratory of Food Nutrition and Safety, Tianjin University of Science and Technology, 1038 Dagu South Road, Tianjin, 300457, China

3 Department of Transfusion, the Fifth Medical Center, Chinese PLA General Hospital, Beijing, 100853, China

\$ Co-first author: Lei Wang, Xiaoyang Yi

* Correspondence authors: Prof. Qiuxia Fu, tiger qhd@sina.com;

Prof. Jun Liang, jliang1118@yeah.net. 


\section{Supporting Figures}

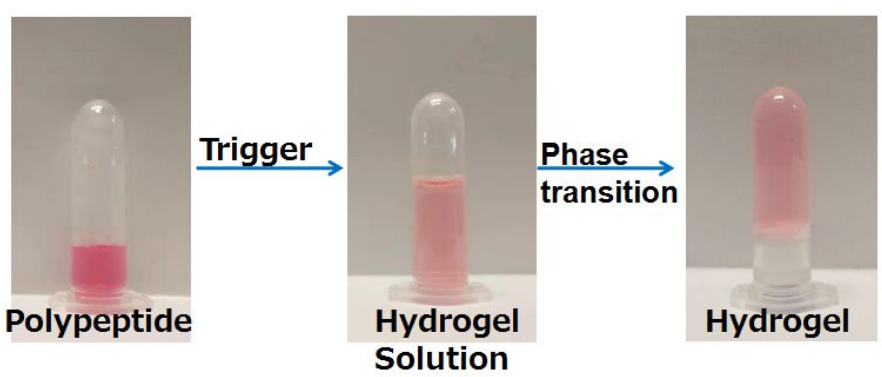

Figure S1. 0.5wt\% peptide responded to form hydrogel initiated by trigger.

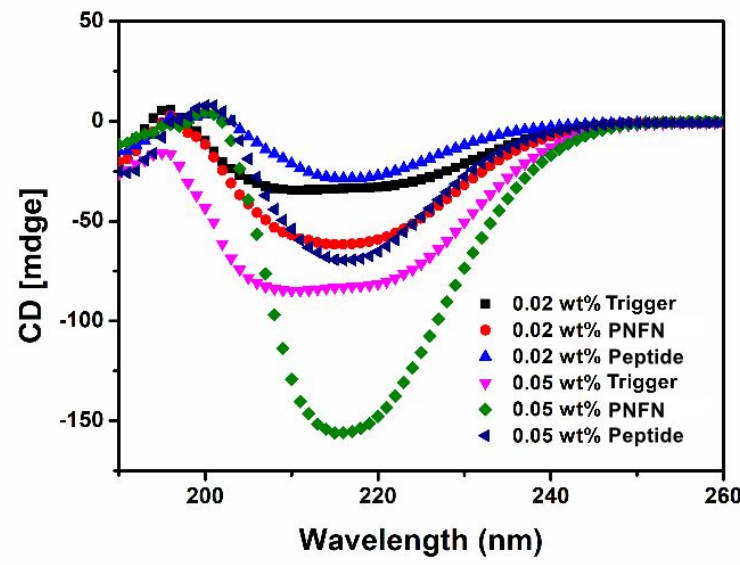

Figure S2. The circular dichroism spectra of trigger, peptide sample and peptide initiated by trigger (PNFN) (at different concentrations of peptide, $\mathrm{c}=0.02 \mathrm{wt} \%$ and $0.05 \mathrm{wt} \%$ ) in a wavelength range from 190 to $260 \mathrm{~nm}$. 